Mridula Garg (Jaipur)

SANGEeta Choudhary (Jaipur)

SARAleEs Nadarajah (Manchester)

\title{
ON THE PRODUCT OF TRIANGULAR RANDOM VARIABLES
}

Abstract. We derive the probability density function (pdf) for the product of three independent triangular random variables. It involves consideration of various cases and subcases. We obtain the pdf for one subcase and present the remaining cases in tabular form. We also indicate how to calculate the pdf for the product of $n$ triangular random variables.

1. Introduction. The triangular distribution is often used when no or little data is available. It is very popular for modelling a subjective estimate of some uncertain quantity in business risk models. One of its earliest applications is to model the average number of defects in a chip (Murphy [11]). It is also used in oil and gas exploration where data is expensive to collect and it is almost impossible to model the population being sampled accurately. The triangular distribution, along with the beta distribution, is also widely used in project management. The symmetric triangular distribution is commonly used in audio dithering, where it is called TPDF (Triangular Probability Density Function). Johnson [7] explores the advantages of using the triangular distribution as a proxy for the beta distribution. Amaral-Turkman and Gonçalves [1] add some new applications of triangular and trapezoidal distributions in the genome analysis, particularly, in the construction of physical mapping of linear and circular chromosomes. Recent popularity of the triangular distribution can be attributed to its use in discrete system simulation [2], Monte Carlo simulation technique [18] and in standard uncertainty analysis software, such as @Risk (developed by the Palisade Corporation) or Crystal Ball (developed by Decision Engineering).

2000 Mathematics Subject Classification: 33C90, 62E99.

Key words and phrases: triangular random variable. 
An extension of the triangular distribution utilized in risk analysis is also discussed by J. Rene Van Dorp and Samuel Kotz [17], with applications in computers and industrial engineering, geotechnical engineering, financial engineering, screening, detection and progression of cancer. Advantages of triangular distribution over beta distribution have been discussed in detail by Kotz and Van Dorp [8].

Products of two or more triangular random variables arise in many situations. Consider the example where triangular distributions model the number of defects in a chip (Murphy [11]). Suppose that an electronic system is made up of $n$ chips and that the numbers of defects in these chips are triangular random variables assumed to be independent. Then the total number of possible failures of the system will be the product of $n$ random variables. Another example is in risk assessment. Many risks can be described by sequences of independent events, say $A_{1}, \ldots, A_{n}$. Suppose that the probability of $A_{i}, i=1, \ldots, n$, is a random variable, with a triangular distribution defined over the unit interval $[0,1]$ (see equation (1) below). Then the probability of the risk occurring will be the product of the $n$ random variables.

The general techniques for determining the distributions of products of random variables are discussed by Donahue [3], Springer and Thompson [16] and Springer [15]. When both random variables follow the gamma, Bessel, Lawrance and Lewis's bivariate exponential, Pearson type VII and the Pareto distribution, the results for the distribution of products have been obtained by Lomnicki [10], Kotz and Srinivasan [9], Nadarajah and Ali [13], Nadarajah and Kotz [14] and Nadarajah [12] respectively. Glen et al. [5] provide a computational algorithm for determining the distribution of the product of two random variables. Glickman and Feng Xu [6] derive the probability density function (pdf) of the product of two triangular random variables.

The aim of this note is to extend the work of Glickman and $\mathrm{Xu}$ [6]. The paper is organized as follows: the pdf for the product of three triangular random variables derived by the use of Mellin transform and its inverse is presented in Section 2. It is assumed that the variables are non-identical and independent. A brief application of this result is discussed in Section 3. Finally, Section 4 outlines the extension to $n$ triangular random variables.

2. PDF of product of three triangular random variables. A random variable $X$ is said to have triangular distribution if it is nonnegative and has continuous probability distribution with lower limit $a_{1}>0$, mode $m_{1}>0$ and upper limit $b_{1}>0$. Its pdf on the support $a_{1} \leq x \leq b_{1}$ is defined as

$$
h^{\prime}(x)= \begin{cases}\frac{2\left(x-a_{1}\right)}{\left(b_{1}-a_{1}\right)\left(m_{1}-a_{1}\right)}, & a_{1} \leq x \leq m_{1}, \\ \frac{2\left(b_{1}-x\right)}{\left(b_{1}-a_{1}\right)\left(b_{1}-m_{1}\right)}, & m_{1} \leq x \leq b_{1} .\end{cases}
$$


We take two more independent and triangularly distributed random variables $Y$ and $Z$ on the supports $a_{2} \leq y \leq b_{2}$ and $a_{3} \leq z \leq b_{3}$, having modes $m_{2}$ and $m_{3}$ respectively. The pdf of the product of three random variables $w=x y z$ can be obtained by using Mellin inversion and is expressed as [16]

$$
h(w)=M_{s}^{-1}\left[M_{s} h^{\prime}(x) M_{s} h^{\prime \prime}(y) M_{s} h^{\prime \prime \prime}(z)\right]
$$

where $h^{\prime}(x), h^{\prime \prime}(y)$ and $h^{\prime \prime \prime}(z)$ denote the pdfs of $X, Y, Z$ respectively. The Mellin transform and its inverse under suitable conditions are defined by

$$
M_{s}(f(x))=\varphi(s)=\int_{0}^{\infty} x^{s-1} f(x) d x
$$

and

$$
M_{s}^{-1}(\varphi(s))=f(x)=\frac{1}{2 \pi \omega} \int_{c-\omega \infty}^{c+\omega \infty} x^{-s} \varphi(s) d s \quad \text { where } \quad \omega=\sqrt{-1}
$$

respectively. The Mellin transform of $h^{\prime}(x)$ can be easily obtained as

(3) $\quad M_{s}\left(h^{\prime}(x)\right)= \begin{cases}\frac{2}{\left(b_{1}-a_{1}\right)\left(m_{1}-a_{1}\right)}\left(\frac{m_{1}^{s+1}}{s+1}-\frac{a_{1} m_{1}^{s}}{s}+\frac{a_{1}^{s+1}}{s(s+1)}\right), & a_{1} \leq x \leq m_{1}, \\ \frac{2}{\left(b_{1}-a_{1}\right)\left(b_{1}-m_{1}\right)}\left(\frac{b_{1}^{s+1}}{s(s+1)}-\frac{b_{1} m_{1}^{s}}{s}+\frac{m_{1}^{s+1}}{s+1}\right), & m_{1} \leq x \leq b_{1} .\end{cases}$

Similarly

(4) $\quad M_{s}\left(h^{\prime \prime}(y)\right)= \begin{cases}\frac{2}{\left(b_{2}-a_{2}\right)\left(m_{2}-a_{2}\right)}\left(\frac{m_{2}^{s+1}}{s+1}-\frac{a_{2} m_{2}^{s}}{s}+\frac{a_{2}^{s+1}}{s(s+1)}\right), & a_{2} \leq y \leq m_{2}, \\ \frac{2}{\left(b_{2}-a_{2}\right)\left(b_{2}-m_{2}\right)}\left(\frac{b_{2}^{s+1}}{s(s+1)}-\frac{b_{2} m_{2}^{s}}{s}+\frac{m_{2}^{s+1}}{s+1}\right), & m_{2} \leq y \leq b_{2},\end{cases}$ and

(5) $\quad M_{s}\left(h^{\prime \prime \prime}(z)\right)= \begin{cases}\frac{2}{\left(b_{3}-a_{3}\right)\left(m_{3}-a_{3}\right)}\left(\frac{m_{3}^{s+1}}{s+1}-\frac{a_{3} m_{3}^{s}}{s}+\frac{a_{3}^{s+1}}{s(s+1)}\right), & a_{3} \leq z \leq m_{3}, \\ \frac{2}{\left(b_{3}-a_{3}\right)\left(b_{3}-m_{3}\right)}\left(\frac{b_{3}^{s+1}}{s(s+1)}-\frac{b_{3} m_{3}^{s}}{s}+\frac{m_{3}^{s+1}}{s+1}\right), & m_{3} \leq z \leq b_{3} .\end{cases}$

Now derivation of the pdf $h(w)$ requires consideration of the following eight different cases where the values of $x, y, z$ are located in different segments:

I. $a_{1} \leq x \leq m_{1}, a_{2} \leq y \leq m_{2}, a_{3} \leq z \leq m_{3}$,

II. $a_{1} \leq x \leq m_{1}, a_{2} \leq y \leq m_{2}, m_{3} \leq z \leq b_{3}$,

III. $a_{1} \leq x \leq m_{1}, m_{2} \leq y \leq b_{2}, a_{3} \leq z \leq m_{3}$,

IV. $a_{1} \leq x \leq m_{1}, m_{2} \leq y \leq b_{2}, m_{3} \leq z \leq b_{3}$,

V. $m_{1} \leq x \leq b_{1}, a_{2} \leq y \leq m_{2}, a_{3} \leq z \leq m_{3}$,

VI. $m_{1} \leq x \leq b_{1}, a_{2} \leq y \leq m_{2}, m_{3} \leq z \leq b_{3}$,

VII. $m_{1} \leq x \leq b_{1}, m_{2} \leq y \leq b_{2}, a_{3} \leq z \leq m_{3}$,

VIII. $m_{1} \leq x \leq b_{1}, m_{2} \leq y \leq b_{2}, m_{3} \leq z \leq b_{3}$. 
2.1. Case I. Let $h_{1}(w)$ be the value of $h(w)$ in Case I. Then using (3)-(5) in equation (2), we get

$$
\begin{aligned}
h_{1}(w)= & M_{s}^{-1}\left[K_{1}\left\{\left(\frac{m_{1}^{s+1}}{s+1}-\frac{a_{1} m_{1}^{s}}{s}+\frac{a_{1}^{s+1}}{s(s+1)}\right)\right\}\right. \\
& \times\left\{\left(\frac{m_{2}^{s+1}}{s+1}-\frac{a_{2} m_{2}^{s}}{s}+\frac{a_{2}^{s+1}}{s(s+1)}\right)\right\} \\
& \left.\times\left\{\left(\frac{m_{3}^{s+1}}{s+1}-\frac{a_{3} m_{3}^{s}}{s}+\frac{a_{3}^{s+1}}{s(s+1)}\right)\right\}\right],
\end{aligned}
$$

where

$$
K_{1}=\frac{8}{\left(b_{1}-a_{1}\right)\left(b_{2}-a_{2}\right)\left(b_{3}-a_{3}\right)\left(m_{1}-a_{1}\right)\left(m_{2}-a_{2}\right)\left(m_{3}-a_{3}\right) .}
$$

Clearly, the right hand side of (6) contains 27 terms. The Mellin inversions of these terms are obtained by partial fractions and using the following known result $[4$, p. $343,(16)]$ :

$$
M_{s}^{-1}\left((s+a)^{-v}\right)=\frac{x^{a}}{\Gamma v}\left(-\ln \frac{x}{a}\right)^{v-1}, \quad \operatorname{Re}(v)>0, \quad \operatorname{Re}(s)>-\operatorname{Re}(a),
$$

with the property $[4$, p. $307,(2)]$

$$
M_{s}^{-1}\left(a^{-s} \varphi(s)\right)=f(a x),
$$

and are given as follows:

$$
\begin{aligned}
M_{s}^{-1} \frac{\left(m_{1} m_{2} m_{3}\right)^{s+1}}{(s+1)^{3}}= & \frac{w}{2}\left(\ln \frac{w}{m_{1} m_{2} m_{3}}\right)^{2}, \quad w<m_{1} m_{2} m_{3}, \\
M_{s}^{-1}\left\{\frac{-m_{1} m_{2} a_{3}\left(m_{1} m_{2} m_{3}\right)^{s}}{s(s+1)^{2}}\right\}= & -m_{1} m_{2} a_{3}\left[1-\frac{w}{m_{1} m_{2} m_{3}}+\frac{w}{m_{1} m_{2} m_{3}} \ln \frac{w}{m_{1} m_{2} m_{3}}\right], \\
M_{s}^{-1}\left\{\frac{\left(m_{1} m_{2} a_{3}\right)^{s+1}}{s(s+1)^{3}}\right\}= & m_{1} m_{2} a_{3}\left[1-\frac{w}{m_{1} m_{2} a_{3}}+\frac{w}{m_{1} m_{2} a_{3}} \ln \frac{w}{m_{1} m_{2} a_{3}}\right. \\
& \left.-\frac{1}{2} \frac{w}{m_{1} m_{2} a_{3}}\left(\ln \frac{w}{m_{1} m_{2} a_{3}}\right)^{2}\right], \quad w<m_{1} m_{2} a_{3}, \\
M_{s}^{-1}\left\{\frac{-m_{1} a_{2} m_{3}\left(m_{1} m_{2} m_{3}\right)^{s}}{s(s+1)^{2}}\right\}= & -m_{1} a_{2} m_{3}\left[1-\frac{w}{m_{1} m_{2} m_{3}}+\frac{w}{m_{1} m_{2} m_{3}} \ln \frac{w}{m_{1} m_{2} m_{3}}\right], \\
M_{s}^{-1}\left\{\frac{m_{1} a_{2} a_{3}\left(m_{1} m_{2} m_{3}\right)^{s}}{s^{2}(s+1)}\right\}= & m_{1} a_{2} a_{3}\left[-1+\frac{w}{m_{1} m_{2} m_{3}}-\ln \frac{w}{m_{1} m_{2} m_{3}}\right], \\
M_{s}^{-1}\left\{\frac{-m_{1} a_{2} a_{3}\left(m_{1} m_{2} a_{3}\right)^{s}}{s^{2}(s+1)^{2}}\right\}= & -m_{1} a_{2} a_{3}\left[-2+\frac{2 w}{m_{1} m_{2} a_{3}}-\ln \frac{w}{m_{1} m_{2} a_{3}},\right. \\
& \left.-\frac{w}{m_{1} m_{2} a_{3}} \ln \frac{w}{m_{1} m_{2} a_{3}}\right], \quad w<m_{1} m_{2} m_{3},
\end{aligned}
$$




$$
\begin{aligned}
& M_{s}^{-1}\left\{\frac{\left(m_{1} a_{2} m_{3}\right)^{s+1}}{s(s+1)^{3}}\right\}=m_{1} a_{2} m_{3}\left[1-\frac{w}{m_{1} a_{2} m_{3}}+\frac{w}{m_{1} a_{2} m_{3}} \ln \frac{w}{m_{1} a_{2} m_{3}}\right. \\
& \left.-\frac{1}{2} \frac{w}{m_{1} a_{2} m_{3}}\left(\ln \frac{w}{m_{1} a_{2} m_{3}}\right)^{2}\right], \quad w<m_{1} a_{2} m_{3}, \\
& M_{s}^{-1}\left\{\frac{-m_{1} a_{2} a_{3}\left(m_{1} m_{2} a_{3}\right)^{s}}{s^{2}(s+1)^{2}}\right\}=-m_{1} a_{2} a_{3}\left[-2+\frac{2 w}{m_{1} a_{2} m_{3}}-\ln \frac{w}{m_{1} a_{2} m_{3}}\right. \\
& \left.-\frac{w}{m_{1} a_{2} m_{3}} \ln \frac{w}{m_{1} a_{2} m_{3}}\right], \quad w<m_{1} a_{2} m_{3}, \\
& M_{s}^{-1}\left\{\frac{\left(m_{1} a_{2} a_{3}\right)^{s+1}}{s^{2}(s+1)^{3}}\right\}=m_{1} a_{2} a_{3}\left[-3+\frac{3 w}{m_{1} a_{2} a_{3}}-\ln \frac{w}{m_{1} a_{2} a_{3}}\right. \\
& \left.-\frac{2 w}{m_{1} a_{2} a_{3}} \ln \frac{w}{m_{1} a_{2} a_{3}}+\frac{1}{2} \frac{w}{m_{1} a_{2} a_{3}}\left(\ln \frac{w}{m_{1} a_{2} a_{3}}\right)^{2}\right] \\
& w<m_{1} a_{2} a_{3} \text {, } \\
& M_{s}^{-1}\left\{\frac{-a_{1} m_{2} m_{3}\left(m_{1} m_{2} m_{3}\right)^{s}}{s(s+1)^{2}}\right\}=-a_{1} m_{2} m_{3}\left[1-\frac{w}{m_{1} m_{2} m_{3}}+\frac{w}{m_{1} m_{2} m_{3}} \ln \frac{w}{m_{1} m_{2} m_{3}}\right], \\
& w<m_{1} m_{2} m_{3}, \\
& M_{s}^{-1}\left\{\frac{a_{1} m_{2} a_{3}\left(m_{1} m_{2} m_{3}\right)^{s}}{s^{2}(s+1)}\right\}=a_{1} m_{2} a_{3}\left[-1+\frac{w}{m_{1} m_{2} m_{3}}-\ln \frac{w}{m_{1} m_{2} m_{3}}\right], \\
& w<m_{1} m_{2} m_{3}, \\
& M_{s}^{-1}\left\{\frac{-a_{1} m_{2} a_{3}\left(m_{1} m_{2} a_{3}\right)^{s}}{s^{2}(s+1)^{2}}\right\}=-a_{1} m_{2} a_{3}\left[-2+\frac{2 w}{m_{1} m_{2} a_{3}}-\ln \frac{w}{m_{1} m_{2} a_{3}}\right. \\
& \left.-\frac{w}{m_{1} m_{2} a_{3}} \ln \frac{w}{m_{1} m_{2} a_{3}}\right], \quad w<m_{1} m_{2} a_{3}, \\
& M_{s}^{-1}\left\{\frac{a_{1} a_{2} m_{3}\left(m_{1} m_{2} m_{3}\right)^{s}}{s^{2}(s+1)}\right\}=a_{1} a_{2} m_{3}\left[-1+\frac{w}{m_{1} m_{2} m_{3}}-\ln \frac{w}{m_{1} m_{2} m_{3}}\right], \\
& M_{s}^{-1}\left\{-a_{1} a_{2} a_{3} \frac{\left(m_{1} m_{2} m_{3}\right)^{s}}{s^{3}}\right\}=-a_{1} a_{2} a_{3}\left[\frac{1}{2}\left(\ln \frac{w}{m_{1} m_{2} m_{3}}\right)^{2}\right], \quad w<m_{1} m_{2} m_{3}, \\
& M_{s}^{-1}\left\{\frac{a_{1} a_{2} a_{3}\left(m_{1} m_{2} a_{3}\right)^{s}}{s^{3}(s+1)}\right\}=a_{1} a_{2} a_{3}\left[1-\frac{w}{m_{1} m_{2} a_{3}}+\ln \frac{w}{m_{1} m_{2} a_{3}}\right. \\
& \left.+\frac{1}{2}\left(\ln \frac{w}{m_{1} m_{2} a_{3}}\right)^{2}\right], \quad w<m_{1} m_{2} a_{3}, \\
& M_{s}^{-1}\left\{\frac{-a_{1} a_{2} m_{3}\left(m_{1} a_{2} m_{3}\right)^{s}}{s^{2}(s+1)^{2}}\right\}=-a_{1} a_{2} m_{3}\left[-2+\frac{2 w}{m_{1} a_{2} m_{3}}-\ln \frac{w}{m_{1} a_{2} m_{3}}\right. \\
& \left.-\frac{w}{m_{1} a_{2} m_{3}} \ln \frac{w}{m_{1} a_{2} m_{3}}\right], \quad w<m_{1} a_{2} m_{3}, \\
& M_{s}^{-1}\left\{\frac{-a_{1} a_{2} a_{3}\left(m_{1} a_{2} m_{3}\right)^{s}}{s^{3}(s+1)}\right\}=a_{1} a_{2} a_{3}\left[1-\frac{w}{m_{1} a_{2} m_{3}}+\ln \frac{w}{m_{1} a_{2} m_{3}}\right. \\
& \left.+\frac{1}{2} \ln \left(\frac{w}{m_{1} a_{2} m_{3}}\right)^{2}\right], \quad w<m_{1} a_{2} m_{3}, \\
& M_{s}^{-1}\left\{\frac{-a_{1} a_{2} a_{3}\left(m_{1} a_{2} a_{3}\right)^{s}}{s^{3}(s+1)^{2}}\right\}=-a_{1} a_{2} a_{3}\left[3-\frac{3 w}{m_{1} a_{2} a_{3}}+2 \ln \frac{w}{m_{1} a_{2} a_{3}}\right. \\
& \left.+\frac{w}{m_{1} a_{2} a_{3}} \ln \frac{w}{m_{1} a_{2} a_{3}}+\frac{1}{2}\left(\ln \frac{w}{m_{1} a_{2} a_{3}}\right)^{2}\right], \\
& w<m_{1} a_{2} a_{3},
\end{aligned}
$$




$$
\begin{aligned}
& M_{s}^{-1}\left\{\frac{\left(a_{1} m_{2} m_{3}\right)^{s+1}}{s(s+1)^{3}}\right\}=a_{1} m_{2} m_{3}\left[1-\frac{w}{a_{1} m_{2} m_{3}}+\frac{w}{a_{1} m_{2} m_{3}} \ln \frac{w}{a_{1} m_{2} m_{3}}\right. \\
& \left.-\frac{1}{2} \frac{w}{a_{1} m_{2} m_{3}}\left(\ln \frac{w}{a_{1} m_{2} m_{3}}\right)^{2}\right], \quad w<a_{1} m_{2} m_{3}, \\
& M_{s}^{-1}\left\{\frac{-a_{1} m_{2} a_{3}\left(a_{1} m_{2} m_{3}\right)^{s}}{s^{2}(s+1)^{2}}\right\}=-a_{1} m_{2} a_{3}\left[-2+\frac{2 w}{a_{1} m_{2} m_{3}}-\ln \frac{w}{a_{1} m_{2} m_{3}}\right. \\
& \left.-\frac{w}{a_{1} m_{2} m_{3}} \ln \frac{w}{a_{1} m_{2} m_{3}}\right], \quad w<a_{1} m_{2} m_{3}, \\
& M_{s}^{-1}\left\{\frac{\left(a_{1} m_{2} a_{3}\right)^{s+1}}{s^{2}(s+1)^{3}}\right\}=a_{1} m_{2} a_{3}\left[-3+\frac{3 w}{a_{1} m_{2} a_{3}}-\ln \frac{w}{a_{1} m_{2} a_{3}}\right. \\
& \left.-\frac{2 w}{a_{1} m_{2} a_{3}} \ln \frac{w}{a_{1} m_{2} a_{3}}+\frac{1}{2} \frac{w}{a_{1} m_{2} a_{3}}\left(\ln \frac{w}{a_{1} m_{2} a_{3}}\right)^{2}\right], \\
& w<a_{1} m_{2} a_{3} \text {, } \\
& M_{s}^{-1}\left\{\frac{-a_{1} a_{2} m_{3}\left(a_{1} m_{2} m_{3}\right)^{s}}{s^{2}(s+1)^{2}}\right\}=-a_{1} a_{2} m_{3}\left[-2+\frac{2 w}{a_{1} m_{2} m_{3}}-\ln \frac{w}{a_{1} m_{2} m_{3}}\right. \\
& \left.-\frac{w}{a_{1} m_{2} m_{3}} \ln \frac{w}{a_{1} m_{2} m_{3}}\right], \quad w<a_{1} m_{2} m_{3}, \\
& M_{s}^{-1}\left\{\frac{a_{1} a_{2} a_{3}\left(a_{1} m_{2} m_{3}\right)^{s}}{s^{3}(s+1)}\right\}=a_{1} a_{2} a_{3}\left[1-\frac{w}{a_{1} m_{2} m_{3}}+\ln \frac{w}{a_{1} m_{2} m_{3}}\right. \\
& \left.+\frac{1}{2}\left(\ln \frac{w}{a_{1} m_{2} m_{3}}\right)^{2}\right], \quad w<a_{1} m_{2} m_{3}, \\
& M_{s}^{-1}\left\{\frac{-a_{1} a_{2} a_{3}\left(a_{1} m_{2} a_{3}\right)^{s}}{s^{3}(s+1)^{2}}\right\}=-a_{1} a_{2} a_{3}\left[3-\frac{3 w}{a_{1} m_{2} a_{3}}+2 \ln \frac{w}{a_{1} m_{2} a_{3}}\right. \\
& \left.+\frac{1}{2}\left(\ln \frac{w}{a_{1} m_{2} a_{3}}\right)^{2}+\frac{w}{a_{1} m_{2} a_{3}} \ln \frac{w}{a_{1} m_{2} a_{3}}\right], \\
& w<a_{1} m_{2} a_{3}, \\
& M_{s}^{-1}\left\{\frac{\left(a_{1} a_{2} m_{3}\right)^{s+1}}{s^{2}(s+1)^{3}}\right\}=a_{1} a_{2} m_{3}\left[-3+\frac{3 w}{a_{1} a_{2} m_{3}}-\ln \frac{w}{a_{1} a_{2} m_{3}}\right. \\
& \left.-\frac{2 w}{a_{1} a_{2} m_{3}} \ln \frac{w}{a_{1} a_{2} m_{3}}+\frac{1}{2} \frac{w}{a_{1} a_{2} m_{3}}\left(\ln \frac{w}{a_{1} a_{2} m_{3}}\right)^{2}\right] \text {, } \\
& w<a_{1} a_{2} m_{3} \text {, } \\
& M_{s}^{-1}\left\{\frac{-a_{1} a_{2} a_{3}\left(a_{1} a_{2} m_{3}\right)^{s}}{s^{3}(s+1)^{2}}\right\}=-a_{1} a_{2} a_{3}\left[3-\frac{3 w}{a_{1} a_{2} m_{3}}+2 \ln \frac{w}{a_{1} a_{2} m_{3}}\right. \\
& \left.+\frac{w}{a_{1} a_{2} m_{3}} \ln \frac{w}{a_{1} a_{2} m_{3}}+\frac{1}{2}\left(\ln \frac{w}{a_{1} a_{2} m_{3}}\right)^{2}\right], \\
& w<a_{1} a_{2} m_{3} \text {, } \\
& M_{s}^{-1}\left\{\frac{\left(a_{1} a_{2} a_{3}\right)^{s+1}}{s^{3}(s+1)^{3}}\right\}=a_{1} a_{2} a_{3}\left[6-\frac{6 w}{a_{1} a_{2} a_{3}}+3 \ln \frac{w}{a_{1} a_{2} a_{3}}+\frac{1}{2}\left(\ln \frac{w}{a_{1} a_{2} a_{3}}\right)^{2}\right. \\
& \left.+\frac{3 w}{a_{1} a_{2} a_{3}} \ln \frac{w}{a_{1} a_{2} a_{3}}-\frac{1}{2} \frac{w}{a_{1} a_{2} a_{3}}\left(\ln \frac{w}{a_{1} a_{2} a_{3}}\right)^{2}\right] \\
& w<a_{1} a_{2} a_{3} .
\end{aligned}
$$

Now with the help of these results, the value of $h_{1}(w)$ as given by (6) can be written for different values of $w$. For example, adding all results for 
$w<m_{1} m_{2} m_{3}$, we get the pdf $h_{1}(w)$ when $w<m_{1} m_{2} m_{3}$ :

$$
\begin{aligned}
& h_{1}(w)=-K_{1}\left[\left(1-\frac{w}{m_{1} m_{2} m_{3}}\right)\right. \\
& \quad \times\left(a_{1} m_{2} m_{3}+m_{1} a_{2} m_{3}+m_{1} m_{2} a_{3}+m_{1} a_{2} a_{3}+a_{1} m_{2} a_{3}+a_{1} a_{2} m_{3}\right) \\
& \quad+\left\{\left(m_{1} a_{2} a_{3}+a_{1} m_{2} a_{3}+a_{1} a_{2} m_{3}\right)\right. \\
& \left.\quad+\left(a_{1} m_{2} m_{3}+m_{1} a_{2} m_{3}+m_{1} m_{2} a_{3}\right) \frac{w}{m_{1} m_{2} m_{3}}\right\} \ln \frac{w}{m_{1} m_{2} m_{3}} \\
& \left.\quad-\frac{1}{2}\left(w-a_{1} a_{2} a_{3}\right)\left(\ln \frac{w}{m_{1} m_{2} m_{3}}\right)^{2}\right]
\end{aligned}
$$

for $w<m_{1} m_{2} m_{3}$.

Proceeding along similar lines we can obtain $h_{1}(w)$ for different values of $w$ as follows:

$$
\begin{aligned}
h_{1}(w) & =K_{1}\left[a_{1}\left(m_{2} m_{3}+2 m_{2} a_{3}+2 a_{2} m_{3}+a_{2} a_{3}\right)\left(1-\frac{w}{a_{1} m_{2} m_{3}}\right)\right. \\
& +\left\{a_{1}\left(m_{2} a_{3}+a_{2} m_{3}+a_{2} a_{3}\right)+\left(m_{2} m_{3}+m_{2} a_{3}+a_{2} m_{3}\right) \frac{w}{m_{2} m_{3}}\right\} \\
& \left.\times \ln \frac{w}{a_{1} m_{2} m_{3}}-\frac{1}{2}\left(w-a_{1} a_{2} a_{3}\right)\left(\ln \frac{w}{a_{1} m_{2} m_{3}}\right)^{2}\right]
\end{aligned}
$$

for $w<a_{1} m_{2} m_{3}$;

$$
\begin{aligned}
h_{1}(w) & =K_{1}\left[a_{2}\left(m_{1} m_{3}+2 m_{1} a_{3}+2 a_{1} m_{3}+a_{1} a_{3}\right)\left(1-\frac{w}{m_{1} a_{2} m_{3}}\right)\right. \\
& +\left\{a_{2}\left(m_{1} a_{3}+a_{1} m_{3}+a_{1} a_{3}\right)+\left(m_{1} m_{3}+m_{1} a_{3}+a_{1} m_{3}\right) \frac{w}{m_{1} m_{3}}\right\} \\
& \left.\times \ln \frac{w}{m_{1} a_{2} m_{3}}-\frac{1}{2}\left(w-a_{1} a_{2} a_{3}\right)\left(\ln \frac{w}{m_{1} a_{2} m_{3}}\right)^{2}\right]
\end{aligned}
$$

for $w<m_{1} a_{2} m_{3}$;

$$
\begin{aligned}
& h_{1}(w)=K_{1}\left[a_{3}\left(m_{1} m_{2}+2 m_{1} a_{2}+2 a_{1} m_{2}+a_{1} a_{2}\right)\left(1-\frac{w}{m_{1} m_{2} a_{3}}\right)\right. \\
& +\left\{a_{3}\left(m_{1} a_{2}+a_{1} m_{2}+a_{1} a_{2}\right)+\left(m_{1} m_{2}+m_{1} a_{2}+a_{1} m_{2}\right) \frac{w}{m_{1} m_{2}}\right\} \\
& \left.\quad \times \ln \frac{w}{m_{1} m_{2} a_{3}}-\frac{1}{2}\left(w-a_{1} a_{2} a_{3}\right)\left(\ln \frac{w}{m_{1} m_{2} a_{3}}\right)^{2}\right]
\end{aligned}
$$

for $w<m_{1} m_{2} a_{3}$; 


$$
\begin{aligned}
h_{1}(w)= & -K_{1}\left[3 a_{1} a_{2}\left(m_{3}+a_{3}\right)\left(1-\frac{w}{a_{1} a_{2} m_{3}}\right)\right. \\
& +\left\{a_{1} a_{2}\left(m_{3}+2 a_{3}\right)+\left(2 m_{3}+a_{3}\right) \frac{w}{m_{3}}\right\} \ln \frac{w}{a_{1} a_{2} m_{3}} \\
& \left.-\frac{1}{2}\left(w-a_{1} a_{2} a_{3}\right)\left(\ln \frac{w}{a_{1} a_{2} m_{3}}\right)^{2}\right]
\end{aligned}
$$

for $w<a_{1} a_{2} m_{3}$;

$$
\begin{aligned}
h_{1}(w)= & -K_{1}\left[3 a_{2} a_{3}\left(a_{1}+m_{1}\right)\left(1-\frac{w}{m_{1} a_{2} a_{3}}\right)\right. \\
& +\left\{a_{2} a_{3}\left(2 a_{1}+m_{1}\right)+\left(a_{1}+2 m_{1}\right) \frac{w}{m_{1}}\right\} \ln \frac{w}{m_{1} a_{2} a_{3}} \\
& \left.-\frac{1}{2}\left(w-a_{1} a_{2} a_{3}\right)\left(\ln \frac{w}{m_{1} a_{2} a_{3}}\right)^{2}\right]
\end{aligned}
$$

for $w<m_{1} a_{2} a_{3}$;

$$
\begin{aligned}
h_{1}(w)= & -K_{1}\left[3 a_{1} a_{3}\left(m_{2}+a_{2}\right)\left(1-\frac{w}{a_{1} m_{2} a_{3}}\right)\right. \\
& +\left\{a_{1} a_{3}\left(m_{2}+2 a_{2}\right)+\left(2 m_{2}+a_{2}\right) \frac{w}{m_{2}}\right\} \ln \frac{w}{a_{1} m_{2} a_{3}} \\
& \left.-\frac{1}{2}\left(w-a_{1} a_{2} a_{3}\right)\left(\ln \frac{w}{a_{1} m_{2} a_{3}}\right)^{2}\right]
\end{aligned}
$$

for $w<a_{1} m_{2} a_{3}$;

$$
\begin{aligned}
h_{1}(w)= & K_{1}\left[6 a_{1} a_{2} a_{3}\left(1-\frac{w}{a_{1} a_{2} a_{3}}\right)+3\left(a_{1} a_{2} a_{3}+w\right) \ln \frac{w}{a_{1} a_{2} a_{3}}\right. \\
& \left.-\frac{1}{2}\left(w-a_{1} a_{2} a_{3}\right)\left(\ln \frac{w}{a_{1} a_{2} a_{3}}\right)^{2}\right]
\end{aligned}
$$

for $w<a_{1} a_{2} a_{3}$.

\section{REMARK.}

1. The equations (8) to (14) can also be obtained from (7) on replacing $m_{1}$ by $a_{1} ; m_{2}$ by $a_{2} ; m_{3}$ by $a_{3} ; m_{1}, m_{2}$ by $a_{1}, a_{2} ; m_{2}, m_{3}$ by $a_{2}, a_{3}$; $m_{1}, m_{3}$ by $a_{1}, a_{3}$; and $m_{1}, m_{2}, m_{3}$ by $a_{1}, a_{2}, a_{3}$, respectively, and each equation is multiplied by the number $(-1)^{\text {no. of replacements }}$.

2. The total number of equations in the case of three variables is $2^{3}$ and it is easily observed that the number of such equations in the case of $n$ variables will be $2^{n}$.

We observe that in Case I, the discussion of the pdf of the product of two triangular random variables $X$ and $Y$ depends upon the relative magnitudes 
of $a_{1} m_{2}$ and $m_{1} a_{2}$. Hence, the pdf of the product $X Y Z$ is first discussed in accordance with the conditions $\left(a_{1} m_{2}<m_{1} a_{2}, a_{1} m_{2}=m_{1} a_{2}, a_{1} m_{2}>\right.$ $\left.m_{1} a_{2}\right)$. If $a_{1} m_{2}<m_{1} a_{2}$ we have the following situation for $w$ :

$$
\left.\begin{array}{c}
a_{1} a_{2} a_{3}<w<a_{1} a_{2} m_{3} \\
a_{1} m_{2} a_{3}<w<a_{1} m_{2} m_{3} \\
m_{1} a_{2} a_{3}<w<m_{1} a_{2} m_{3} \\
m_{1} m_{2} a_{3}<w<m_{1} m_{2} m_{3}
\end{array}\right\}
$$

This situation will further depend upon the relative magnitudes of $a_{2} m_{3}$, $m_{2} a_{3}, a_{1} m_{2} m_{3}, m_{1} a_{2} a_{3}$ for which we have the following nine possibilities termed as subcases:

(i) $a_{1} a_{2} m_{3}<a_{1} m_{2} a_{3}, \quad a_{1} m_{2} m_{3}<m_{1} a_{2} a_{3}, \quad m_{1} a_{2} m_{3}<m_{1} m_{2} a_{3}$,

(ii) $a_{1} a_{2} m_{3}<a_{1} m_{2} a_{3}, \quad a_{1} m_{2} m_{3}=m_{1} a_{2} a_{3}, m_{1} a_{2} m_{3}<m_{1} m_{2} a_{3}$,

(iii) $a_{1} a_{2} m_{3}<a_{1} m_{2} a_{3}, \quad a_{1} m_{2} m_{3}>m_{1} a_{2} a_{3}, \quad m_{1} a_{2} m_{3}<m_{1} m_{2} a_{3}$,

(iv) $a_{1} a_{2} m_{3}=a_{1} m_{2} a_{3}, \quad a_{1} m_{2} m_{3}<m_{1} a_{2} a_{3}, \quad m_{1} a_{2} m_{3}=m_{1} m_{2} a_{3}$,

(v) $a_{1} a_{2} m_{3}=a_{1} m_{2} a_{3}, \quad a_{1} m_{2} m_{3}=m_{1} a_{2} a_{3}, \quad m_{1} a_{2} m_{3}=m_{1} m_{2} a_{3}$,

(vi) $a_{1} a_{2} m_{3}=a_{1} m_{2} a_{3}, \quad a_{1} m_{2} m_{3}>m_{1} a_{2} a_{3}, \quad m_{1} a_{2} m_{3}=m_{1} m_{2} a_{3}$,

(vii) $a_{1} a_{2} m_{3}>a_{1} m_{2} a_{3}, \quad a_{1} m_{2} m_{3}<m_{1} a_{2} a_{3}, \quad m_{1} a_{2} m_{3}>m_{1} m_{2} a_{3}$,

(viii) $a_{1} a_{2} m_{3}>a_{1} m_{2} a_{3}, \quad a_{1} m_{2} m_{3}=m_{1} a_{2} a_{3}, \quad m_{1} a_{2} m_{3}>m_{1} m_{2} a_{3}$,

(ix) $a_{1} a_{2} m_{3}>a_{1} m_{2} a_{3}, \quad a_{1} m_{2} m_{3}>m_{1} a_{2} a_{3}, \quad m_{1} a_{2} m_{3}>m_{1} m_{2} a_{3}$.

Further, if $a_{1} m_{2}=m_{1} a_{2}$ then

$$
\left.\begin{array}{rl}
a_{1} a_{2} a_{3} & <w<a_{1} a_{2} m_{3} \\
a_{1} m_{2} a_{3} & <w<a_{1} m_{2} m_{3} \\
m_{1} m_{2} a_{3} & <w<m_{1} m_{2} m_{3}
\end{array}\right\}
$$

and the corresponding subcases will be

(x) $a_{1} a_{2} m_{3}<a_{1} m_{2} a_{3}, \quad a_{1} m_{2} m_{3}<m_{1} m_{2} a_{3}$,

(xi) $a_{1} a_{2} m_{3}<a_{1} m_{2} a_{3}, \quad a_{1} m_{2} m_{3}=m_{1} m_{2} a_{3}$,

(xii) $a_{1} a_{2} m_{3}<a_{1} m_{2} a_{3}, \quad a_{1} m_{2} m_{3}>m_{1} m_{2} a_{3}$,

xiii) $a_{1} a_{2} m_{3}=a_{1} m_{2} a_{3}, \quad a_{1} m_{2} m_{3}<m_{1} m_{2} a_{3}$,

(xiv) $a_{1} a_{2} m_{3}=a_{1} m_{2} a_{3}, \quad a_{1} m_{2} m_{3}=m_{1} m_{2} a_{3}$,

(xv) $a_{1} a_{2} m_{3}=a_{1} m_{2} a_{3}, \quad a_{1} m_{2} m_{3}>m_{1} m_{2} a_{3}$,

(xvi) $a_{1} a_{2} m_{3}>a_{1} m_{2} a_{3}, \quad a_{1} m_{2} m_{3}<m_{1} m_{2} a_{3}$,

(xvii) $a_{1} a_{2} m_{3}>a_{1} m_{2} a_{3}, \quad a_{1} m_{2} m_{3}=m_{1} m_{2} a_{3}$,

(xviii) $a_{1} a_{2} m_{3}>a_{1} m_{2} a_{3}, \quad a_{1} m_{2} m_{3}>m_{1} m_{2} a_{3}$.

Next, if $a_{1} m_{2}>m_{1} a_{2}$, then

$$
\left.\begin{array}{rl}
a_{1} a_{2} a_{3} & <w<a_{1} a_{2} m_{3} \\
m_{1} a_{2} a_{3} & <w<m_{1} a_{2} m_{3} \\
a_{1} m_{2} a_{3} & <w<a_{1} m_{2} m_{3} \\
m_{1} m_{2} a_{3} & <w<m_{1} m_{2} m_{3}
\end{array}\right\}
$$


and the subcases are

(xix) $a_{1} a_{2} m_{3}<m_{1} a_{2} a_{3}, \quad m_{1} a_{2} m_{3}<a_{1} m_{2} a_{3}, \quad a_{1} m_{2} m_{3}<m_{1} m_{2} a_{3}$,

(xx) $a_{1} a_{2} m_{3}<m_{1} a_{2} a_{3}, \quad m_{1} a_{2} m_{3}=a_{1} m_{2} a_{3}, \quad a_{1} m_{2} m_{3}<m_{1} m_{2} a_{3}$,

(xxi) $a_{1} a_{2} m_{3}<m_{1} a_{2} a_{3}, \quad m_{1} a_{2} m_{3}>a_{1} m_{2} a_{3}, \quad a_{1} m_{2} m_{3}<m_{1} m_{2} a_{3}$,

(xxii) $a_{1} a_{2} m_{3}=m_{1} a_{2} a_{3}, \quad m_{1} a_{2} m_{3}<a_{1} m_{2} a_{3}, \quad a_{1} m_{2} m_{3}=m_{1} m_{2} a_{3}$,

(xxiii) $a_{1} a_{2} m_{3}=m_{1} a_{2} a_{3}, \quad m_{1} a_{2} m_{3}=a_{1} m_{2} a_{3}, \quad a_{1} m_{2} m_{3}=m_{1} m_{2} a_{3}$,

(xxiv) $a_{1} a_{2} m_{3}=m_{1} a_{2} a_{3}, \quad m_{1} a_{2} m_{3}>a_{1} m_{2} a_{3}, \quad a_{1} m_{2} m_{3}=m_{1} m_{2} a_{3}$,

(xxv) $a_{1} a_{2} m_{3}>m_{1} a_{2} a_{3}, \quad m_{1} a_{2} m_{3}<a_{1} m_{2} a_{3}, \quad a_{1} m_{2} m_{3}>m_{1} m_{2} a_{3}$,

(xxvi) $a_{1} a_{2} m_{3}>m_{1} a_{2} a_{3}, \quad m_{1} a_{2} m_{3}=a_{1} m_{2} a_{3}, \quad a_{1} m_{2} m_{3}>m_{1} m_{2} a_{3}$,

(xxvii) $a_{1} a_{2} m_{3}>m_{1} a_{2} a_{3}, \quad m_{1} a_{2} m_{3}>a_{1} m_{2} a_{3}, \quad a_{1} m_{2} m_{3}>m_{1} m_{2} a_{3}$.

2.2. Evaluation of pdf for a particular subcase. To find the value of $h_{1}(w)$ for subcases (i) to (xxvii), we will require the following equations which are combinations of equations (7) to (13) and give pdf for different intervals of $w$ :

$$
\begin{aligned}
& h_{1}(w)=K_{1}\left[-6 a_{1} a_{2} a_{3}+6 w-\left(3 w+3 a_{1} a_{2} a_{3}\right) \ln \frac{w}{a_{1} a_{2} a_{3}}\right. \\
& \left.+\frac{1}{2}\left(w-a_{1} a_{2} a_{3}\right)\left(\ln \frac{w}{a_{1} a_{2} a_{3}}\right)^{2}\right] \text {, } \\
& h_{1}(w)=K_{1}\left[\left(3 a_{1} a_{2} m_{3}-3 a_{1} a_{2} a_{3}\right)-w\left(-3+\frac{3 a_{3}}{m_{3}}\right)\right. \\
& -\left(w+a_{1} a_{2} a_{3}\right)\left(\ln \frac{w}{a_{1} a_{2} a_{3}}-2 \ln \frac{a_{3}}{m_{3}}\right)+\left(\frac{w a_{3}}{m_{3}}+a_{1} a_{2} m_{3}\right) \ln \frac{w}{a_{1} a_{2} m_{3}} \\
& +\frac{1}{2}\left(w-a_{1} a_{2} a_{3}\right)\left\{\left(\ln \frac{w}{m_{1} m_{2} m_{3}}\right)^{2}-\left(\ln \frac{w}{m_{1} m_{2} a_{3}}\right)^{2}\right. \\
& +\left(\ln \frac{w}{m_{1} a_{2} a_{3}}\right)^{2}-\left(\ln \frac{w}{m_{1} a_{2} m_{3}}\right)^{2}+\left(\ln \frac{w}{a_{1} m_{2} a_{3}}\right)^{2} \\
& \left.\left.-\left(\ln \frac{w}{a_{1} m_{2} m_{3}}\right)^{2}\right\}\right] \text {, } \\
& h_{1}(w)=K_{1}\left[3\left(a_{1} m_{2} a_{3}+a_{1} a_{2} m_{3}\right)-3 w\left(\frac{a_{2}}{m_{2}}+\frac{a_{3}}{m_{3}}\right)\right. \\
& +\left(w+a_{1} a_{2} a_{3}\right)\left(\ln \frac{w}{a_{1} m_{2} m_{3}}+\ln \frac{a_{2} a_{3}}{m_{2} m_{3}}\right) \\
& +\left(\frac{w a_{2}}{m_{2}}+a_{1} m_{2} a_{3}\right) \ln \frac{w}{a_{1} m_{2} a_{3}}+\left(\frac{w a_{3}}{m_{3}}+a_{1} a_{2} m_{3}\right) \ln \frac{w}{a_{1} a_{2} m_{3}} \\
& +\frac{1}{2}\left(w-a_{1} a_{2} a_{3}\right)\left\{\left(\ln \frac{w}{m_{1} m_{2} m_{3}}\right)^{2}-\left(\ln \frac{w}{m_{1} m_{2} a_{3}}\right)^{2}\right. \\
& \left.\left.+\left(\ln \frac{w}{m_{1} a_{2} a_{3}}\right)^{2}-\left(\ln \frac{w}{m_{1} a_{2} m_{3}}\right)^{2}-\left(\ln \frac{w}{a_{1} m_{2} m_{3}}\right)^{2}\right\}\right],
\end{aligned}
$$


(18)

$$
\begin{aligned}
h_{1}(w)= & K_{1}\left[\left(a_{1} a_{2} m_{3}+a_{1} m_{2} a_{3}-a_{1} m_{2} m_{3}-a_{1} a_{2} a_{3}\right)\right. \\
& -w\left(-1+\frac{a_{2}}{m_{2}}+\frac{a_{3}}{m_{3}}-\frac{a_{2} a_{3}}{m_{2} m_{3}}\right)+\left(w+a_{1} a_{2} a_{3}\right)\left(\ln \frac{a_{2} a_{3}}{m_{2} m_{3}}\right) \\
& -\left(\frac{w a_{2}}{m_{2}}+a_{1} m_{2} a_{3}\right) \ln \frac{a_{3}}{m_{3}}-\left(\frac{w a_{3}}{m_{3}}+a_{1} a_{2} m_{3}\right) \ln \frac{a_{2}}{m_{2}} \\
& +\frac{1}{2}\left(w-a_{1} a_{2} a_{3}\right)\left\{\left(\ln \frac{w}{m_{1} m_{2} m_{3}}\right)^{2}-\left(\ln \frac{w}{m_{1} m_{2} a_{3}}\right)^{2}\right. \\
& \left.\left.+\left(\ln \frac{w}{m_{1} a_{2} a_{3}}\right)^{2}-\left(\ln \frac{w}{m_{1} a_{2} m_{3}}\right)^{2}\right\}\right]
\end{aligned}
$$

$$
\begin{aligned}
h_{1}(w)= & K_{1}\left[\left(3 m_{1} a_{2} a_{3}+a_{1} m_{2} a_{3}+a_{1} a_{2} m_{3}-a_{1} m_{2} m_{3}+2 a_{1} a_{2} a_{3}\right)\right. \\
& -w\left(2+\frac{3 a_{1}}{m_{1}}+\frac{a_{2}}{m_{2}} \frac{a_{3}}{m_{3}}-\frac{a_{2} a_{3}}{m_{2} m_{3}}\right) \\
& +\left(w+a_{1} a_{2} a_{3}\right)\left(\ln \frac{w}{m_{1} a_{2} m_{3}}+\ln \frac{w}{m_{1} m_{2} a_{3}}\right) \\
& +\left(\frac{w a_{1}}{m_{1}}+m_{1} a_{2} a_{3}\right) \ln \frac{w}{m_{1} a_{2} a_{3}}-\left(\frac{w a_{2}}{m_{2}}+a_{1} m_{2} a_{3}\right) \ln \frac{a_{3}}{m_{3}} \\
& -\left(\frac{w a_{3}}{m_{3}}+a_{1} a_{2} m_{3}\right) \ln \frac{a_{2}}{m_{2}} \\
& +\frac{1}{2}\left(w-a_{1} a_{2} a_{3}\right)\left\{\left(\ln \frac{w}{m_{1} m_{2} m_{3}}\right)^{2}-\left(\ln \frac{w}{m_{1} m_{2} a_{3}}\right)^{2}\right. \\
& \left.\left.-\left(\ln \frac{w}{m_{1} a_{2} m_{3}}\right)^{2}\right\}\right],
\end{aligned}
$$

(20) $\quad h_{1}(w)=K_{1}\left[\left(m_{1} a_{2} a_{3}+a_{1} m_{2} a_{3}-a_{1} a_{2} m_{3}-m_{1} a_{2} m_{3}-a_{1} m_{2} m_{3}\right.\right.$

$$
\begin{aligned}
& \left.+a_{1} a_{2} a_{3}\right)-w\left(1+\frac{a_{1}}{m_{1}}+\frac{a_{2}}{m_{2}}-\frac{a_{3}}{m_{3}}-\frac{a_{2} a_{3}}{m_{2} m_{3}}-\frac{a_{1} a_{3}}{m_{1} m_{3}}\right) \\
& +\left(w+a_{1} a_{2} a_{3}\right)\left(\ln \frac{w}{m_{1} m_{2} a_{3}}\right) \\
& -\left(\frac{w a_{1}}{m_{1}}+\frac{w a_{2}}{m_{2}}+m_{1} a_{2} a_{3}+a_{1} m_{2} a_{3}\right) \ln \frac{a_{3}}{m_{3}} \\
& \left.\left.\left.-\left(\frac{w a_{3}}{m_{3}}+a_{1} a_{2} m_{3}\right) \ln \frac{w}{m_{1} m_{2} m_{3}}\right)^{2}-\left(\ln \frac{w}{m_{1} m_{2} a_{3}}\right)^{2}\right\}\right],
\end{aligned}
$$


(21)

$$
\begin{aligned}
h_{1}(w) & =K_{1}\left[\left(-a_{1} m_{2} m_{3}-m_{1} a_{2} m_{3}-m_{1} m_{2} a_{3}-a_{1} a_{2} m_{3}-m_{1} a_{2} a_{3}\right.\right. \\
& \left.-a_{1} m_{2} a_{3}\right)+w\left(\frac{a_{1}}{m_{1}}+\frac{a_{2}}{m_{2}}+\frac{a_{3}}{m_{3}}+\frac{a_{1} a_{2}}{m_{1} m_{2}}+\frac{a_{2} a_{3}}{m_{2} m_{3}}+\frac{a_{1} a_{3}}{m_{1} m_{3}}\right) \\
& -\left(\frac{w a_{1}}{m_{1}}+\frac{w a_{2}}{m_{2}}+\frac{w a_{3}}{m_{3}}+a_{1} a_{2} m_{3}+a_{1} m_{2} a_{3}+m_{1} a_{2} a_{3}\right) \\
& \left.\times \ln \frac{w}{m_{1} m_{2} m_{3}}+\frac{1}{2}\left(w-a_{1} a_{2} a_{3}\right)\left(\ln \frac{w}{m_{1} m_{2} m_{3}}\right)^{2}\right],
\end{aligned}
$$

(22) $\quad h_{1}(w)=K_{1}\left[\left(3 m_{1} a_{2} a_{3}+3 a_{1} m_{2} a_{3}+3 a_{1} a_{2} m_{3}+3 a_{1} a_{2} a_{3}\right)\right.$

$$
-w\left(3+\frac{3 a_{1}}{m_{1}}+\frac{3 a_{2}}{m_{2}}+\frac{3 a_{3}}{m_{3}}\right)
$$$$
+\left(w+a_{1} a_{2} a_{3}\right)\left(\ln \frac{w}{a_{1} a_{2} a_{3}}+2 \ln \frac{w}{m_{1} m_{2} m_{3}}\right)
$$$$
+\left(\frac{w a_{1}}{m_{1}}+m_{1} a_{2} a_{3}\right) \ln \frac{w}{m_{1} a_{2} a_{3}}
$$$$
+\left(\frac{w a_{2}}{m_{2}}+a_{1} m_{2} a_{3}\right) \ln \frac{w}{a_{1} m_{2} a_{3}}
$$$$
+\left(\frac{w a_{3}}{m_{3}}+a_{1} a_{2} m_{3}\right) \ln \frac{w}{a_{1} a_{2} m_{3}}
$$$$
+\frac{1}{2}\left(w-a_{1} a_{2} a_{3}\right)\left\{\left(\ln \frac{w}{m_{1} m_{2} m_{3}}\right)^{2}-\left(\ln \frac{w}{a_{1} m_{2} m_{3}}\right)^{2}\right.
$$

$$
\begin{aligned}
& \left.\left.-\left(\ln \frac{w}{m_{1} a_{2} m_{3}}\right)^{2}-\left(\ln \frac{w}{m_{1} m_{2} a_{3}}\right)^{2}\right\}\right] \\
h_{1}(w)=K_{1} & {\left[\left(3 a_{1} m_{2} a_{3}-3 a_{1} a_{2} a_{3}\right)+w\left(3-\frac{3 a_{2}}{m_{2}}\right)\right.} \\
& -\left(w+a_{1} a_{2} a_{3}\right)\left(\ln \frac{w}{a_{1} a_{2} a_{3}}+2 \ln \frac{m_{2}}{a_{2}}\right) \\
& +\left(\frac{w a_{2}}{m_{2}}+a_{1} m_{2} a_{3}\right) \ln \frac{w}{a_{1} m_{2} a_{3}} \\
+ & \frac{1}{2}\left(w-a_{1} a_{2} a_{3}\right)\left\{\left(\ln \frac{w}{m_{1} m_{2} m_{3}}\right)^{2}-\left(\ln \frac{w}{a_{1} m_{2} m_{3}}\right)^{2}\right. \\
& -\left(\ln \frac{w}{m_{1} a_{2} m_{3}}\right)^{2}-\left(\ln \frac{w}{m_{1} m_{2} a_{3}}\right)^{2}+\left(\ln \frac{w}{m_{1} a_{2} a_{3}}\right)^{2} \\
& \left.\left.+\left(\ln \frac{w}{a_{1} a_{2} m_{3}}\right)^{2}\right\}\right]
\end{aligned}
$$


(24)

$$
\begin{aligned}
h_{1}(w)= & K_{1}\left[\left(m_{1} a_{2} a_{3}+a_{1} a_{2} a_{3}+a_{1} a_{2} m_{3}-m_{1} m_{2} a_{3}-a_{1} m_{2} m_{3}\right.\right. \\
& \left.-a_{1} m_{2} a_{3}\right)+w\left(-1-\frac{a_{1}}{m_{1}}+\frac{a_{2}}{m_{2}}-\frac{a_{3}}{m_{3}}+\frac{a_{2} a_{3}}{m_{2} m_{3}}+\frac{a_{1} a_{2}}{m_{1} m_{2}}\right) \\
& +\left(w+a_{1} a_{2} a_{3}\right)\left(\ln \frac{w}{m_{1} a_{2} m_{3}}\right) \\
& -\left(\frac{w a_{2}}{m_{2}}+a_{1} m_{2} a_{3}\right) \ln \frac{w}{m_{1} m_{2} m_{3}} \\
& +\left(\frac{w a_{3}}{m_{3}}+a_{1} a_{2} m_{3}\right) \ln \frac{m_{2}}{a_{2}}+\left(\frac{w a_{1}}{m_{1}}+m_{1} a_{2} a_{3}\right) \ln \frac{m_{2}}{a_{2}} \\
& \left.+\frac{1}{2}\left(w-a_{1} a_{2} a_{3}\right)\left\{\left(\ln \frac{w}{m_{1} m_{2} m_{3}}\right)^{2}-\left(\ln \frac{w}{m_{1} a_{2} m_{3}}\right)^{2}\right\}\right]
\end{aligned}
$$

$$
\begin{aligned}
h_{1}(w)= & K_{1}\left[\left(a_{1} m_{2} a_{3}+2 a_{1} a_{2} a_{3}+3 a_{1} a_{2} m_{3}+m_{1} a_{2} a_{3}-m_{1} m_{2} a_{3}\right)\right. \\
& -w\left(2+\frac{a_{1}}{m_{1}}+\frac{a_{2}}{m_{2}}+\frac{3 a_{3}}{m_{3}}-\frac{a_{1} a_{2}}{m_{1} m_{2}}\right) \\
& +\left(w+a_{1} a_{2} a_{3}\right)\left(\ln \frac{w}{a_{1} m_{2} m_{3}}+\ln \frac{w}{m_{1} a_{2} m_{3}}\right) \\
& +\left(\frac{w a_{2}}{m_{2}}+a_{1} m_{2} a_{3}\right) \ln \frac{m_{1}}{a_{1}}+\left(\frac{w a_{1}}{m_{1}}+m_{1} a_{2} a_{3}\right) \ln \frac{m_{2}}{a_{2}} \\
& +\left(\frac{w a_{3}}{m_{3}}+a_{1} a_{2} m_{3}\right) \ln \frac{w}{a_{1} a_{2} m_{3}} \\
& +\frac{1}{2}\left(w-a_{1} a_{2} a_{3}\right)\left\{\left(\ln \frac{w}{m_{1} m_{2} m_{3}}\right)^{2}-\left(\ln \frac{w}{a_{1} m_{2} m_{3}}\right)^{2}\right. \\
& \left.\left.-\left(\ln \frac{w}{m_{1} a_{2} m_{3}}\right)^{2}\right\}\right],
\end{aligned}
$$

(26) $\quad h_{1}(w)=K_{1}\left[\left(3 a_{1} m_{2} a_{3}+3 m_{1} a_{2} a_{3}\right)-w\left(\frac{3 a_{1}}{m_{1}}+\frac{3 a_{2}}{m_{2}}\right)\right.$

$$
\begin{aligned}
& +\left(w+a_{1} a_{2} a_{3}\right)\left(\ln \frac{w}{m_{1} m_{2} a_{3}}+\ln \frac{a_{1} a_{2}}{m_{1} m_{2}}\right) \\
& +\left(\frac{w a_{1}}{m_{1}}+m_{1} a_{2} a_{3}\right) \ln \frac{w}{m_{1} a_{2} a_{3}}+\left(\frac{w a_{2}}{m_{2}}+a_{1} m_{2} a_{3}\right) \ln \frac{w}{a_{1} m_{2} a_{3}} \\
& +\frac{1}{2}\left(w-a_{1} a_{2} a_{3}\right)\left\{\left(\ln \frac{w}{m_{1} m_{2} m_{3}}\right)^{2}-\left(\ln \frac{w}{a_{1} m_{2} m_{3}}\right)^{2}\right. \\
& \left.\left.-\left(\ln \frac{w}{m_{1} a_{2} m_{3}}\right)^{2}-\left(\ln \frac{w}{m_{1} m_{2} a_{3}}\right)^{2}+\left(\ln \frac{w}{a_{1} a_{2} m_{3}}\right)^{2}\right\}\right]
\end{aligned}
$$




$$
\begin{aligned}
h_{1}(w)= & K_{1}\left[\left(3 m_{1} a_{2} a_{3}+3 a_{1} a_{2} m_{3}\right)-w\left(\frac{3 a_{1}}{m_{1}}+\frac{3 a_{3}}{m_{3}}\right)\right. \\
& +\left(w+a_{1} a_{2} a_{3}\right)\left(\ln \frac{w}{m_{1} a_{2} m_{3}}+\ln \frac{a_{1} a_{3}}{m_{1} m_{3}}\right) \\
& +\left(\frac{w a_{1}}{m_{1}}+m_{1} a_{2} m_{3}\right) \ln \frac{w}{m_{1} a_{2} m_{3}} \\
& +\left(\frac{w a_{3}}{m_{3}}+a_{1} a_{2} m_{3}\right) \ln \frac{w}{a_{1} a_{2} m_{3}} \\
& +\frac{1}{2}\left(w-a_{1} a_{2} a_{3}\right)\left\{\left(\ln \frac{w}{m_{1} m_{2} m_{3}}\right)^{2}-\left(\ln \frac{w}{a_{1} m_{2} m_{3}}\right)^{2}\right. \\
& \left.\left.-\left(\ln \frac{w}{m_{1} a_{2} m_{3}}\right)^{2}-\left(\ln \frac{w}{m_{1} m_{2} a_{3}}\right)^{2}+\left(\ln \frac{w}{a_{1} m_{2} a_{3}}\right)^{2}\right\}\right]
\end{aligned}
$$

(28)

$$
\begin{aligned}
h_{1}(w)= & K_{1}\left[\left(a_{1} a_{2} m_{3}-a_{1} a_{2} a_{3}+m_{1} a_{2} a_{3}-m_{1} a_{2} m_{3}\right)\right. \\
& +w\left(1-\frac{a_{1}}{m_{1}}-\frac{a_{3}}{m_{3}}+\frac{a_{1} a_{3}}{m_{1} m_{3}}\right)+\left(w+a_{1} a_{2} a_{3}\right)\left(\ln \frac{a_{1} a_{3}}{m_{1} m_{3}}\right) \\
& +\left(\frac{w a_{1}}{m_{1}}+m_{1} a_{2} a_{3}\right) \ln \frac{m_{3}}{a_{3}}+\left(\frac{w a_{3}}{m_{3}}+a_{1} a_{2} m_{3}\right) \ln \frac{m_{1}}{a_{1}} \\
& +\frac{1}{2}\left(w-a_{1} a_{2} a_{3}\right)\left\{\left(\ln \frac{w}{m_{1} m_{2} m_{3}}\right)^{2}-\left(\ln \frac{w}{a_{1} m_{2} m_{3}}\right)^{2}\right. \\
& \left.\left.-\left(\ln \frac{w}{m_{1} m_{2} a_{3}}\right)^{2}+\left(\ln \frac{w}{a_{1} m_{2} a_{3}}\right)^{2}\right\}\right]
\end{aligned}
$$

(29) $h_{1}(w)=K_{1}\left[\left(a_{1} a_{2} m_{3}+2 a_{1} a_{2} a_{3}+3 a_{1} m_{2} a_{3}+m_{1} a_{2} a_{3}-m_{1} a_{2} m_{3}\right)\right.$

$$
\begin{aligned}
& +w\left(-2-\frac{a_{1}}{m_{1}}-\frac{3 a_{2}}{m_{2}}-\frac{a_{3}}{m_{3}}+\frac{a_{1} a_{3}}{m_{1} m_{3}}\right) \\
& +\left(w+a_{1} a_{2} a_{3}\right)\left(\ln \frac{w}{a_{1} m_{2} m_{3}}+\ln \frac{w}{m_{1} m_{2} a_{3}}\right)
\end{aligned}
$$$$
\times\left(\frac{w a_{1}}{m_{1}}+m_{1} a_{2} a_{3}\right) \ln \frac{m_{3}}{a_{3}}+\left(\frac{w a_{2}}{m_{2}}+a_{1} m_{2} a_{3}\right) \ln \frac{w}{a_{1} m_{2} a_{3}}
$$$$
+\left(\frac{w a_{3}}{m_{3}}+a_{1} a_{2} m_{3}\right) \ln \frac{m_{1}}{a_{1}}
$$$$
+\frac{1}{2}\left(w-a_{1} a_{2} a_{3}\right)\left\{\left(\ln \frac{w}{m_{1} m_{2} m_{3}}\right)^{2}-\left(\ln \frac{w}{a_{1} m_{2} m_{3}}\right)^{2}\right.
$$$$
\left.\left.-\left(\ln \frac{w}{m_{1} m_{2} a_{3}}\right)^{2}\right\}\right]
$$ 


$$
\begin{aligned}
& \text { (30) } \quad h_{1}(w)=K_{1}\left[\left(3 m_{1} a_{2} a_{3}-3 a_{1} a_{2} a_{3}\right)+w\left(3-\frac{3 a_{1}}{m_{1}}\right)\right. \\
& +\left(w+a_{1} a_{2} a_{3}\right)\left(-\ln \frac{w}{a_{1} a_{2} a_{3}}-2 \ln \frac{m_{1}}{a_{1}}\right)+\left(\frac{w a_{1}}{m_{1}}+m_{1} a_{2} a_{3}\right) \ln \frac{w}{m_{1} a_{2} a_{3}}
\end{aligned}
$$$$
+\frac{1}{2}\left(w-a_{1} a_{2} a_{3}\right)\left\{\left(\ln \frac{w}{m_{1} m_{2} m_{3}}\right)^{2}-\left(\ln \frac{w}{a_{1} m_{2} m_{3}}\right)^{2}\right.
$$

$$
\begin{aligned}
\left.\left.-\left(\ln \frac{w}{m_{1} a_{2} m_{3}}\right)^{2}-\left(\ln \frac{w}{m_{1} m_{2} a_{3}}\right)^{2}+\left(\ln \frac{w}{a_{1} a_{2} m_{3}}\right)^{2}+\left(\ln \frac{w}{a_{1} m_{2} a_{3}}\right)^{2}\right\}\right] \\
(31) \quad h_{1}(w)=K_{1}\left[\left(a_{1} m_{2} a_{3}+a_{1} a_{2} m_{3}+a_{1} a_{2} a_{3}-m_{1} m_{2} a_{3}-m_{1} a_{2} m_{3}\right.\right. \\
\left.-m_{1} a_{2} a_{3}\right)+w\left(-1+\frac{a_{1}}{m_{1}}-\frac{a_{2}}{m_{2}}-\frac{a_{3}}{m_{3}}+\frac{a_{1} a_{2}}{m_{1} m_{2}}+\frac{a_{1} a_{3}}{m_{1} m_{3}}\right) \\
+\left(w+a_{1} a_{2} a_{3}\right)\left(\ln \frac{w}{a_{1} m_{2} m_{3}}\right)-\left(\frac{w a_{1}}{m_{1}}+m_{1} a_{2} a_{3}\right) \ln \frac{w}{m_{1} m_{2} m_{3}} \\
+\left(\frac{w a_{2}}{m_{2}}+a_{1} m_{2} a_{3}\right) \ln \frac{m_{1}}{a_{1}}+\left(\frac{w a_{3}}{m_{3}}+a_{1} a_{2} m_{3}\right) \ln \frac{m_{1}}{a_{1}} \\
\left.+\frac{1}{2}\left(w-a_{1} a_{2} a_{3}\right)\left\{\left(\ln \frac{w}{m_{1} m_{2} m_{3}}\right)^{2}-\left(\ln \frac{w}{a_{1} m_{2} m_{3}}\right)^{2}\right\}\right]
\end{aligned}
$$

Under conditions (A) and its subcase (i), i.e. $\left(a_{1} a_{2} m_{3}<a_{1} m_{2} a_{3}, a_{1} m_{2} m_{3}<\right.$ $\left.m_{1} a_{2} a_{3}, m_{1} a_{2} m_{3}<m_{1} m_{2} a_{3}\right)$ we observe that $w$ is defined for seven different intervals which are $\left(a_{1} a_{2} a_{3}, a_{1} a_{2} m_{3}\right),\left(a_{1} a_{2} m_{3}, a_{1} m_{2} a_{3}\right),\left(a_{1} m_{2} a_{3}\right.$, $\left.a_{1} m_{2} m_{3}\right),\left(a_{1} m_{2} m_{3}, m_{1} a_{2} a_{3}\right),\left(m_{1} a_{2} a_{3}, m_{1} a_{2} m_{3}\right),\left(m_{1} a_{2} m_{3}, m_{1} m_{2} a_{3}\right)$, and $\left(m_{1} m_{2} a_{3}, m_{1} m_{2} m_{3}\right)$. Also, $a_{1} a_{2} m_{3}<\left(a_{1} m_{2} a_{3}, a_{1} m_{2} m_{3}, m_{1} a_{2} a_{3}, m_{1} a_{2} m_{3}\right.$, $\left.m_{1} m_{2} a_{3}, m_{1} m_{2} m_{3}\right)$ and for the first interval we have $w<a_{1} a_{2} m_{3}$, thus the pdf for this interval is obtained by adding equations (7) to (13) and is given in (15). Similarly, the pdf for the remaining six intervals can be calculated and are given by eqs. (16) to (21), respectively, and represented in Table 1.

Table 1. Sequence of points determining intervals for $w$ and pdf for respective intervals (Case I)

\begin{tabular}{|l|l|l|}
\hline Subcase & Sequence of points determining intervals for $w$ & $\begin{array}{l}\text { Equations giving } \\
\text { pdf for respective } \\
\text { interval }\end{array}$ \\
\hline (i) & $\begin{array}{l}a_{1} a_{2} a_{3}<a_{1} a_{2} m_{3}<a_{1} m_{2} a_{3}<a_{1} m_{2} m_{3}<m_{1} a_{2} a_{3}< \\
m_{1} a_{2} m_{3}<m_{1} m_{2} a_{3}<m_{1} m_{2} m_{3}\end{array}$ & $\begin{array}{l}(15),(16),(17), \\
(18),(19),(20),(21)\end{array}$ \\
\hline (ii) & $\begin{array}{l}a_{1} a_{2} a_{3}<a_{1} a_{2} m_{3}<a_{1} m_{2} a_{3}<a_{1} m_{2} m_{3}=m_{1} a_{2} a_{3}< \\
m_{1} a_{2} m_{3}<m_{1} m_{2} a_{3}<m_{1} m_{2} m_{3}\end{array}$ & $\begin{array}{l}(15),(16),(17), \\
(19),(20),(21)\end{array}$ \\
\hline (iii) & $\begin{array}{l}a_{1} a_{2} a_{3}<a_{1} a_{2} m_{3}<a_{1} m_{2} a_{3}<m_{1} a_{2} a_{3}<a_{1} m_{2} m_{3}< \\
m_{1} a_{2} m_{3}<m_{1} m_{2} a_{3}<m_{1} m_{2} m_{3}\end{array}$ & $\begin{array}{l}(15),(16),(17), \\
(22),(19),(20),(21)\end{array}$ \\
\hline
\end{tabular}




\begin{tabular}{|c|c|c|}
\hline (iv) & $\begin{array}{l}a_{1} a_{2} a_{3}<a_{1} a_{2} m_{3}=a_{1} m_{2} a_{3}<a_{1} m_{2} m_{3}<m_{1} a_{2} a_{3}< \\
m_{1} a_{2} m_{3}=m_{1} m_{2} a_{3}<m_{1} m_{2} m_{3}\end{array}$ & $\begin{array}{l}(15),(16),(18), \\
(19),(21)\end{array}$ \\
\hline (v) & $\begin{array}{l}a_{1} a_{2} a_{3}<a_{1} a_{2} m_{3}=a_{1} m_{2} a_{3}<a_{1} m_{2} m_{3}=m_{1} a_{2} a_{3}< \\
m_{1} a_{2} m_{3}=m_{1} m_{2} a_{3}<m_{1} m_{2} m_{3}\end{array}$ & $(15),(17),(19),(21)$ \\
\hline (vi) & $\begin{array}{l}a_{1} a_{2} a_{3}<a_{1} a_{2} m_{3}=a_{1} m_{2} a_{3}<m_{1} a_{2} a_{3}<a_{1} m_{2} m_{3}< \\
m_{1} a_{2} m_{3}=m_{1} m_{2} a_{3}<m_{1} m_{2} m_{3}\end{array}$ & $\begin{array}{l}(15),(17),(22), \\
(19),(21)\end{array}$ \\
\hline (vii) & $\begin{array}{l}a_{1} a_{2} a_{3}<a_{1} m_{2} a_{3}<a_{1} a_{2} m_{3}<a_{1} m_{2} m_{3}<m_{1} a_{2} a_{3}< \\
m_{1} m_{2} a_{3}<m_{1} a_{2} m_{3}<m_{1} m_{2} m_{3}\end{array}$ & $\begin{array}{l}(15),(23),(17) \\
(18),(19),(24),(21)\end{array}$ \\
\hline (viii) & $\begin{array}{l}a_{1} a_{2} a_{3}<a_{1} m_{2} a_{3}<a_{1} a_{2} m_{3}<a_{1} m_{2} m_{3}=m_{1} a_{2} a_{3}< \\
m_{1} m_{2} a_{3}<m_{1} a_{2} m_{3}<m_{1} m_{2} m_{3}\end{array}$ & $\begin{array}{l}(15),(23),(17), \\
(19),(24),(21)\end{array}$ \\
\hline (ix) & $\begin{array}{l}a_{1} a_{2} a_{3}<a_{1} m_{2} a_{3}<a_{1} a_{2} m_{3}<m_{1} a_{2} a_{3}<a_{1} m_{2} m_{3}< \\
m_{1} m_{2} a_{3}<m_{1} a_{2} m_{3}<m_{1} m_{2} m_{3}\end{array}$ & $\begin{array}{l}(15),(23),(17) \\
(22),(19),(24),(21)\end{array}$ \\
\hline (x) & $\begin{array}{l}a_{1} a_{2} a_{3}<a_{1} a_{2} m_{3}<a_{1} m_{2} a_{3}<a_{1} m_{2} m_{3}<m_{1} m_{2} a_{3}< \\
m_{1} m_{2} m_{3}\end{array}$ & $\begin{array}{l}(15),(16),(22), \\
(20),(21)\end{array}$ \\
\hline (xi) & $\begin{array}{l}a_{1} a_{2} a_{3}<a_{1} a_{2} m_{3}<a_{1} m_{2} a_{3}<a_{1} m_{2} m_{3}=m_{1} m_{2} a_{3}< \\
m_{1} m_{2} m_{3}\end{array}$ & $(15),(16),(22),(21)$ \\
\hline (xii) & $\begin{array}{l}a_{1} a_{2} a_{3}<a_{1} a_{2} m_{3}<a_{1} m_{2} a_{3}<m_{1} m_{2} a_{3}<a_{1} m_{2} m_{3}< \\
m_{1} m_{2} m_{3}\end{array}$ & $\begin{array}{l}(15),(16),(22), \\
(25),(21)\end{array}$ \\
\hline (xiii) & $\begin{array}{l}a_{1} a_{2} a_{3}<a_{1} a_{2} m_{3}=a_{1} m_{2} a_{3}<a_{1} m_{2} m_{3}<m_{1} m_{2} a_{3}< \\
m_{1} m_{2} m_{3}\end{array}$ & $(15),(22),(20),(21)$ \\
\hline (xiv) & $\begin{array}{l}a_{1} a_{2} a_{3}<a_{1} a_{2} m_{3}=a_{1} m_{2} a_{3}<a_{1} m_{2} m_{3}=m_{1} m_{2} a_{3}< \\
m_{1} m_{2} m_{3}\end{array}$ & $(15),(22),(21)$ \\
\hline$(\mathrm{xv})$ & $\begin{array}{l}a_{1} a_{2} a_{3}<a_{1} a_{2} m_{3}=a_{1} m_{2} a_{3}<m_{1} m_{2} a_{3}<a_{1} m_{2} m_{3}< \\
m_{1} m_{2} m_{3}\end{array}$ & $(15),(22),(25),(21)$ \\
\hline (xvi) & $\begin{array}{l}a_{1} a_{2} a_{3}<a_{1} m_{2} a_{3}<a_{1} a_{2} m_{3}<a_{1} m_{2} m_{3}<m_{1} m_{2} a_{3}< \\
m_{1} m_{2} m_{3}\end{array}$ & $\begin{array}{l}(15),(26),(22), \\
(20),(21)\end{array}$ \\
\hline (xvii) & $\begin{array}{l}a_{1} a_{2} a_{3}<a_{1} m_{2} a_{3}<a_{1} a_{2} m_{3}<a_{1} m_{2} m_{3}=m_{1} m_{2} a_{3}< \\
m_{1} m_{2} m_{3}\end{array}$ & $(15),(26),(22),(21)$ \\
\hline (xviii) & $\begin{array}{l}a_{1} a_{2} a_{3}<a_{1} m_{2} a_{3}<a_{1} a_{2} m_{3}<m_{1} m_{2} a_{3}<a_{1} m_{2} m_{3}< \\
m_{1} m_{2} m_{3}\end{array}$ & $\begin{array}{l}(15),(26),(22), \\
(25),(21)\end{array}$ \\
\hline (xix) & $\begin{array}{l}a_{1} a_{2} a_{3}<a_{1} a_{2} m_{3}<m_{1} a_{2} a_{3}<m_{1} a_{2} m_{3}<a_{1} m_{2} a_{3}< \\
a_{1} m_{2} m_{3}<m_{1} m_{2} a_{3}<m_{1} m_{2} m_{3}\end{array}$ & $\begin{array}{l}(15),(16),(27) \\
(28),(29),(20),(21)\end{array}$ \\
\hline$(\mathrm{xx})$ & $\begin{array}{l}a_{1} a_{2} a_{3}<a_{1} a_{2} m_{3}<m_{1} a_{2} a_{3}<m_{1} a_{2} m_{3}=a_{1} m_{2} a_{3}< \\
a_{1} m_{2} m_{3}<m_{1} m_{2} a_{3}<m_{1} m_{2} m_{3}\end{array}$ & $\begin{array}{l}(15),(16),(27), \\
(29),(20),(21)\end{array}$ \\
\hline$(\mathrm{xxi})$ & $\begin{array}{l}a_{1} a_{2} a_{3}<a_{1} a_{2} m_{3}<m_{1} a_{2} a_{3}<a_{1} m_{2} a_{3}<m_{1} a_{2} m_{3}< \\
a_{1} m_{2} m_{3}<m_{1} m_{2} a_{3}<m_{1} m_{2} m_{3}\end{array}$ & $\begin{array}{l}(15),(16),(27) \\
(22),(29),(20),(21)\end{array}$ \\
\hline (xxii) & $\begin{array}{l}a_{1} a_{2} a_{3}<a_{1} a_{2} m_{3}=m_{1} a_{2} a_{3}<m_{1} a_{2} m_{3}<a_{1} m_{2} a_{3}< \\
a_{1} m_{2} m_{3}=m_{1} m_{2} a_{3}<m_{1} m_{2} m_{3}\end{array}$ & $\begin{array}{l}(15),(27),(28), \\
(29),(21)\end{array}$ \\
\hline (xxiii) & $\begin{array}{l}a_{1} a_{2} a_{3}<a_{1} a_{2} m_{3}=m_{1} a_{2} a_{3}<m_{1} a_{2} m_{3}=a_{1} m_{2} a_{3}< \\
a_{1} m_{2} m_{3}=m_{1} m_{2} a_{3}<m_{1} m_{2} m_{3}\end{array}$ & $(15),(27),(29),(21)$ \\
\hline (xxiv) & $\begin{array}{l}a_{1} a_{2} a_{3}<a_{1} a_{2} m_{3}=m_{1} a_{2} a_{3}<a_{1} m_{2} a_{3}<m_{1} a_{2} m_{3}< \\
a_{1} m_{2} m_{3}=m_{1} m_{2} a_{3}<m_{1} m_{2} m_{3}\end{array}$ & $\begin{array}{l}(15),(27),(22), \\
(29),(21)\end{array}$ \\
\hline
\end{tabular}




\begin{tabular}{|l|l|l|}
\hline (xxv) & $\begin{array}{l}a_{1} a_{2} a_{3}<m_{1} a_{2} a_{3}<a_{1} a_{2} m_{3}<m_{1} a_{2} m_{3}<a_{1} m_{2} a_{3}< \\
m_{1} m_{2} a_{3}<a_{1} m_{2} m_{3}<m_{1} m_{2} m_{3}\end{array}$ & $(15),(30),(27)$, \\
& $(28),(29),(31),(21)$ \\
\hline (xxvi) & $a_{1} a_{2} a_{3}<m_{1} a_{2} a_{3}<a_{1} a_{2} m_{3}<m_{1} a_{2} m_{3}=a_{1} m_{2} a_{3}<$ & $(15),(30),(27)$, \\
& $m_{1} m_{2} a_{3}<a_{1} m_{2} m_{3}<m_{1} m_{2} m_{3}$ & $(29),(31),(21)$ \\
\hline (xxvii) & $a_{1} a_{2} a_{3}<m_{1} a_{2} a_{3}<a_{1} a_{2} m_{3}<a_{1} m_{2} a_{3}<m_{1} a_{2} m_{3}<$ & $(15),(30),(27)$, \\
& $m_{1} m_{2} a_{3}<a_{1} m_{2} m_{3}<m_{1} m_{2} m_{3}$ & $(22),(29),(31),(21)$ \\
\hline
\end{tabular}

Table 1 shows the sequence of points determining intervals for $w$ and pdf for corresponding intervals for all the subcases of Case I. This completes the discussion of Case I. It is easy to observe that Cases II to VIII can be handled by making the changes in Case I as indicated in Table 2, where

$$
\begin{aligned}
K_{1} & =\frac{8}{\prod_{i=1}^{3}\left(b_{i}-a_{i}\right) \prod_{j=1}^{3}\left(m_{j}-a_{j}\right)}, & K_{5} & =\frac{m_{1}-a_{1}}{b_{1}-m_{1}} K_{1}, \\
K_{2} & =\frac{m_{3}-a_{3}}{b_{3}-m_{3}} K_{1}, & K_{6} & =\prod_{j=1,3} \frac{m_{j}-a_{j}}{b_{j}-m_{j}} K_{1}, \\
K_{3} & =\frac{m_{2}-a_{2}}{b_{2}-m_{2}} K_{1}, & K_{7} & =\prod_{j=1,2} \frac{m_{j}-a_{j}}{b_{j}-m_{j}} K_{1}, \\
K_{4} & =\prod_{j=2,3} \frac{m_{j}-a_{j}}{b_{j}-m_{j}} K_{1}, & K_{8} & =\frac{8}{\prod_{i=1}^{3}\left(b_{i}-a_{i}\right) \prod_{j=1}^{3}\left(b_{j}-m_{j}\right)} .
\end{aligned}
$$

Table 2. Changes involved in the discussion of Cases II to VIII

\begin{tabular}{|l|l|l|l|}
\hline Case & Pdf & Eqs. $(7)$ to $(14)$ & Subcases (i) to (xxvii) \\
\hline II & $h_{2}(w)$ & $a_{3} \rightarrow b_{3}, K_{1} \rightarrow K_{2}$ & $a_{3} \rightarrow m_{3}, m_{3} \rightarrow b_{3}$ \\
\hline $\mathrm{III}$ & $h_{3}(w)$ & $a_{2} \rightarrow b_{2}, K_{1} \rightarrow K_{3}$ & $a_{2} \rightarrow m_{2}, m_{2} \rightarrow b_{2}$ \\
\hline $\mathrm{IV}$ & $h_{4}(w)$ & $a_{2} \rightarrow b_{2}, a_{3} \rightarrow b_{3}, K_{1} \rightarrow K_{4}$ & $a_{2} \rightarrow m_{2}, a_{3} \rightarrow m_{3}, m_{2} \rightarrow b_{2}, m_{3} \rightarrow b_{3}$ \\
\hline $\mathrm{V}$ & $h_{5}(w)$ & $a_{1} \rightarrow b_{1}, K_{1} \rightarrow K_{5}$ & $a_{1} \rightarrow m_{1}, m_{1} \rightarrow b_{1}$ \\
\hline $\mathrm{VI}$ & $h_{6}(w)$ & $a_{1} \rightarrow b_{1}, a_{3} \rightarrow b_{3}, K_{1} \rightarrow K_{6}$ & $a_{1} \rightarrow m_{1}, a_{3} \rightarrow m_{3}, m_{1} \rightarrow b_{1}, m_{3} \rightarrow b_{3}$ \\
\hline $\mathrm{VII}$ & $h_{7}(w)$ & $a_{1} \rightarrow b_{1}, a_{2} \rightarrow b_{2}, K_{1} \rightarrow K_{7}$ & $a_{1} \rightarrow m_{1}, a_{2} \rightarrow m_{2}, m_{1} \rightarrow b_{1}, m_{2} \rightarrow b_{2}$ \\
\hline $\mathrm{VIII}$ & $h_{8}(w)$ & $\begin{array}{l}a_{1} \rightarrow b_{1}, a_{2} \rightarrow b_{2}, a_{3} \rightarrow b_{3}, \\
K_{1} \rightarrow K_{8}\end{array}$ & $\begin{array}{l}a_{1} \rightarrow m_{1}, a_{2} \rightarrow m_{2}, a_{3} \rightarrow m_{3}, \\
m_{1} \rightarrow b_{1}, m_{2} \rightarrow b_{2}, m_{3} \rightarrow b_{3}\end{array}$ \\
\hline
\end{tabular}

3. Application. Here, we return to the example discussed in Section 1 motivated by Murphy [11]. Suppose that the functioning of an electronic system is determined independently by $n$ chips it has. Suppose too that the number of defects in each of the $n$ chips has the triangular distribution with $a=0, b=4$ and $m=2$, the values used in Murphy [11]. The total number of 


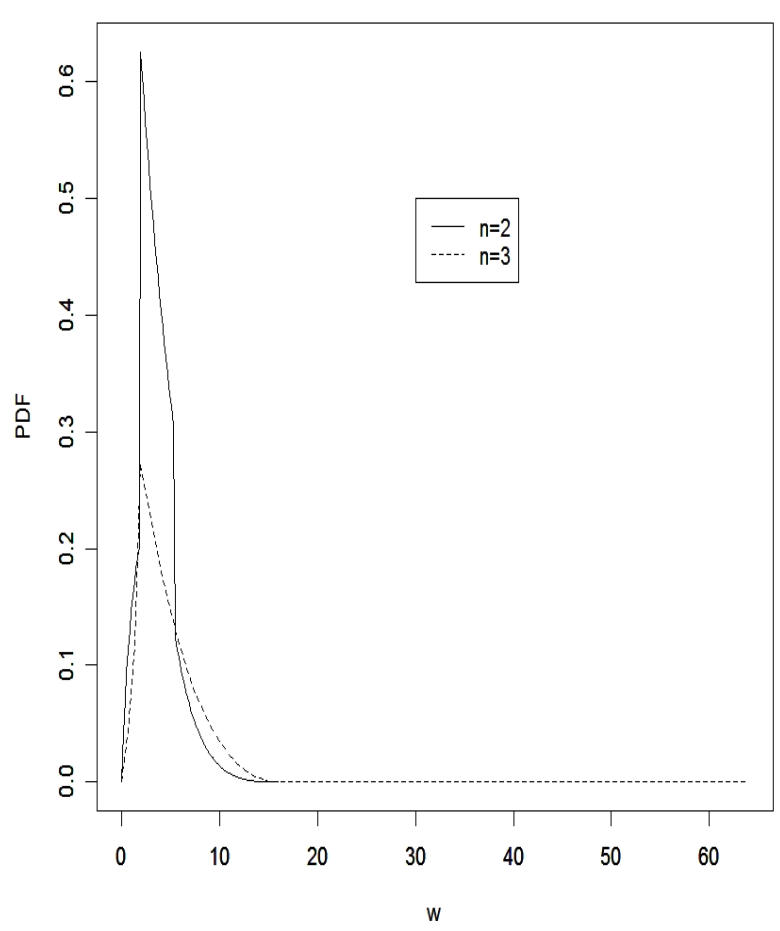

Fig. 1. Pdf of the total number of possible failures of the electronic system for $n=2$ and $n=3$

possible failures of the system is then a product of $n$ independent triangular random variables. The pdf of this total number is shown in Figure 1 for $n=2$ and $n=3$. Figures of this kind can be used to obtain summary measures and for quality control purposes.

4. Generalization for $n$ variables. Now, we give the total number of cases and their subcases to be considered when the number of random variables is $n$.

For cases: We see that the number of cases to be considered for the product of two triangular random variables is $2^{2}$ and for three $2^{3}$. So it is easy to see that in the case of $n$ variables there will be $2^{n}$ cases. We observe that equation (7) plays a major role in the discussion of Case I; we give below its general form when the number of random variables is $n \geq 2$ :

$$
\begin{aligned}
& h(w)=-\left(A_{1}+{ }^{n-2} C_{1} A_{2}+{ }^{n-2} C_{2} A_{3}+\cdots+A_{n-1}\right) \\
& +\left[\left\{\left(A_{1}+{ }^{n-2} C_{1} A_{2}+{ }^{n-2} C_{2} A_{3}+\cdots+A_{n-1}\right)\right.\right. \\
& -\left(A_{1}+{ }^{n-3} C_{1} A_{2}+\cdots+A_{n-2}\right) \ln \frac{w}{A}
\end{aligned}
$$




$$
\begin{aligned}
& +\frac{1}{2 !}\left(A_{1}+{ }^{n-4} C_{1} A_{2}+\cdots+A_{n-3}\right)\left(\ln \frac{w}{A}\right)^{2}-\cdots \\
& \left.\left.+(-1)^{n} \frac{1}{(n-2) !} A_{1}\left(\ln \frac{w}{A}\right)^{n-2}\right\}+(-1)^{n-1} \frac{1}{(n-1) !} A\left(\ln \frac{w}{A}\right)^{n-1}\right] \frac{w}{A} \\
& -\left[\left(A_{2}+{ }^{n-3} C_{1} A_{3}+{ }^{n-3} C_{2} A_{4}+\cdots+A_{n-1}\right)\left(\ln \frac{w}{A}\right)\right. \\
& +\frac{1}{2 !}\left(A_{3}+{ }^{n-4} C_{1} A_{4}+\cdots+A_{n-1}\right)\left(\ln \frac{w}{A}\right)^{2}+\cdots \\
& \left.+\frac{1}{(n-2) !} A_{n-1}\left(\ln \frac{w}{A}\right)^{n-2}+\frac{1}{(n-1) !} A_{n}\left(\ln \frac{w}{A}\right)^{n-1}\right] \quad(w<A)
\end{aligned}
$$

where $A=m_{1} \ldots m_{n}$ and $A_{k}(k=1, \ldots, n)$ is the sum of all terms obtained on replacing in $A$ the quantities $m_{i_{1}}, \ldots, m_{i_{k}}$ by $a_{i_{1}}, \ldots, a_{i_{k}}$, respectively, for $i_{1}, \ldots, i_{k} \in\{1, \ldots, n\}$ with $i_{1}<\cdots<i_{k}$. The other $2^{n}-1$ equations, which correspond to equations (8) to (14) of Case I, can be obtained on generalizing Remark 1 to $n$ variables.

For subcases: When the number of r.v. is two, we have the three subcases $\left(a_{1} m_{2}<m_{1} a_{2}, a_{1} m_{2}=m_{1} a_{2}, a_{1} m_{2}>m_{1} a_{2}\right)$ for each case. When the number of r.v. is three we find that each subcase gives rise to $3^{2}$ cases. Thus the total number of subcases becomes $3^{1} * 3^{2}=3^{3}=27$. If we further observe the pattern we find that in the case of four variables each subcase will give rise to $3^{3}$ cases. So the total number of subcases is $3^{1} * 3^{2} * 3^{3}=3^{6}$. Similarly for $n$ random variables the number of subcases is

$$
3^{1} * \cdots * 3^{n-1}=3^{(n-1) n / 2} .
$$

Figure 2 shows the enumeration of subcases in Case I for four variables $X$, $Y, Z$ and $U$ with the supports $a_{1} \leq x \leq b_{1}, a_{2} \leq y \leq b_{2}, a_{3} \leq z \leq b_{3}$, $a_{4} \leq u \leq b_{4}$ and modes $m_{1}, m_{2}, m_{3}$ and $m_{4}$, respectively. The following is a generalization of (A) for four variables:

$$
\left.\begin{array}{c}
a_{1} a_{2} a_{3} a_{4}<w<a_{1} a_{2} a_{3} m_{4} \\
a_{1} a_{2} m_{3} a_{4}<w<a_{1} a_{2} m_{3} m_{4} \\
a_{1} m_{2} a_{3} a_{4}<w<a_{1} m_{2} a_{3} m_{4} \\
a_{1} m_{2} m_{3} a_{4}<w<a_{1} m_{2} m_{3} m_{4} \\
m_{1} a_{2} a_{3} a_{4}<w<m_{1} a_{2} a_{3} m_{4} \\
m_{1} a_{2} m_{3} a_{4}<w<m_{1} a_{2} m_{3} m_{4} \\
m_{1} m_{2} a_{3} a_{4}<w<m_{1} m_{2} a_{3} m_{4} \\
m_{1} m_{2} m_{3} a_{4}<w<m_{1} m_{2} m_{3} m_{4}
\end{array}\right\}
$$




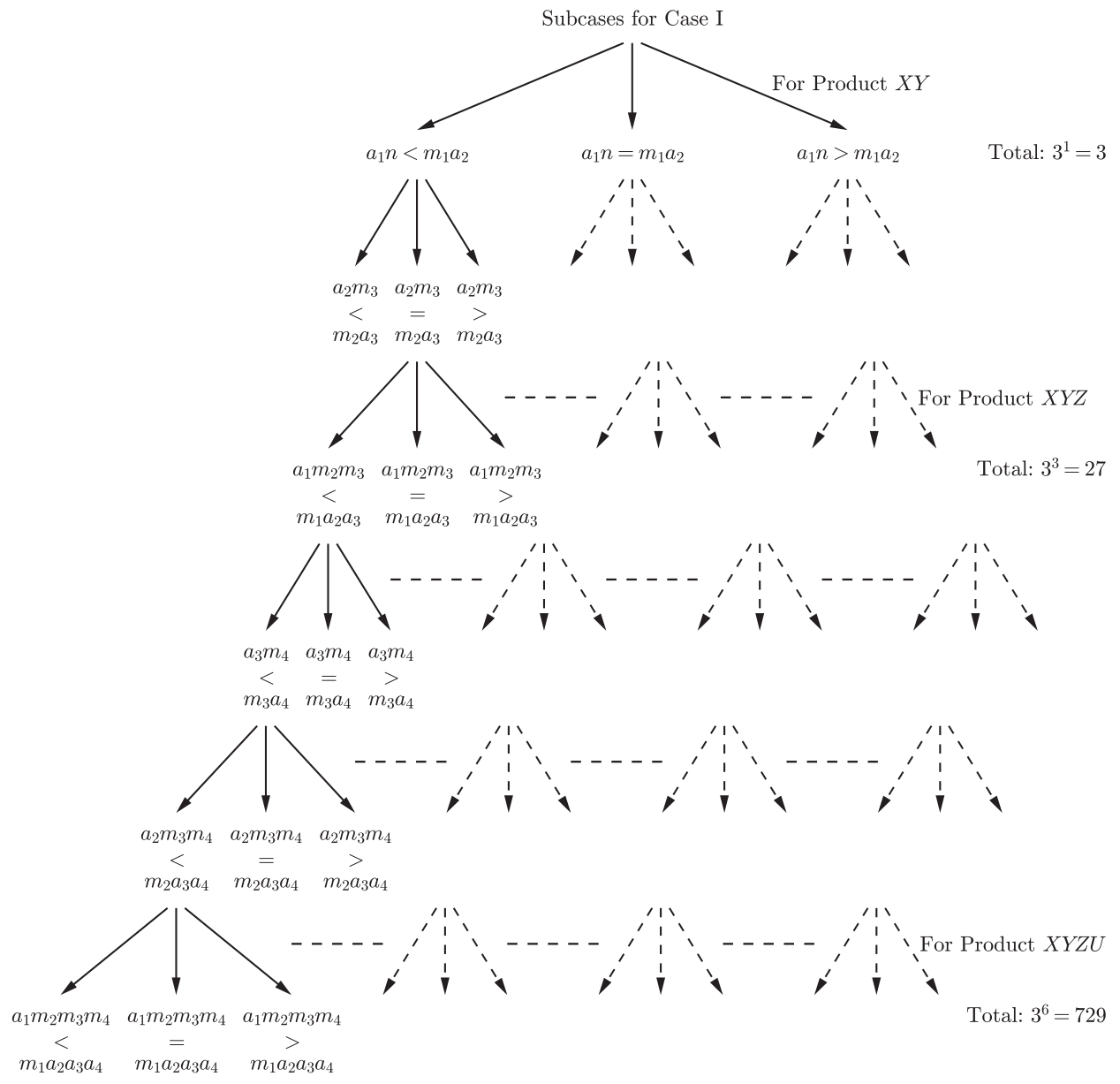

Fig. 2. Enumeration of subcases in Case I for four variables

Acknowledgements. The first author is grateful to University Grants Commission, Bhopal, for providing necessary financial assistance to carry out the present work.

All of the authors would like to thank the Editor and the referee for carefully reading the paper and for their comments which greatly improved the paper.

\section{References}

[1] M. A. Amaral-Turkman and L. Gonçalves, Triangular and trapezoidal distributions: Applications in the genome analysis, J. Statist. Theory Practice 2 (2008), 45-54.

[2] J. Banks, J. S. Carson, B. L. Nelson and D. M. Nicol, Discrete-Event System Simulation, 3rd ed., Prentice-Hall, Upper Saddle River, NJ, 2001. 
[3] J. D. Donahue, Products and quotients of random variables and their applications, ARL 64-115, Aerospace Research Laboratories, Wright-Patterson Air Force Base, $\mathrm{OH}, 1964$.

[4] A. Erdélyi et al., Tables of Integral Transforms, Vol. 1, McGraw-Hill, New York, 1954.

[5] A. G. Glen, L. M. Leemis and J. H. Drew, Computing the distribution of the product of two continuous random variables, Comput. Statist. Data Anal. 44 (2004), 451464.

[6] T. S. Glickman and F. Xu, The distribution of the product of two triangular random variables, Statist. Probab. Lett. 78 (2008), 2821-2826.

[7] D. Johnson, The triangular distribution as a proxy for the beta distribution in risk analysis, The Statistician 46 (1997), 387-398.

[8] S. Kotz and J. R. van Dorp, Beyond Beta: Other Continuous Families of Distributions with Bounded Support and Applications, World Sci., Singapore, 2004.

[9] S. Kotz and R. Srinivasan, Distribution of product and quotient of Bessel function variates, Ann. Inst. Statist. Math. 21 (1969), 201-210.

[10] Z. A. Lomnicki, On the distribution of products of random variables, J. Roy. Statist. Soc. Ser. B 29 (1967), 513-524.

[11] B. T. Murphy, Cost-size optima of monolithic integrated circuits, Proc. IEEE 52 (1964), 1537-1545.

[12] S. Nadarajah, On the product of generalized Pareto random variables, Appl. Econom. Lett. 15 (2008), 253-259.

[13] S. Nadarajah and M. M. Ali, The distribution of sums, products and ratios for Lawrance and Lewis's bivariate exponential random variables, Comput. Statist. Data Anal. 50 (2006), 3449-3463.

[14] S. Nadarajah and S. Kotz, On the product XY for the elliptically symmetric Pearson type VII distribution, Math. Proc. Roy. Irish Acad. 106A (2006), 149-162.

[15] M. D. Springer, The Algebra of Random Variables, Wiley, New York, 1979.

[16] M. D. Springer and W. E. Thompson, The distribution of products of independent random variables, SIAM J. Appl. Math. 14 (1966), 511-526.

[17] J. van Dorp and S. Kotz, A novel extension of the triangular distribution and its parameter estimation, The Statistician 51 (2002), 63-79.

[18] J. F. Wright, Monte Carlo Risk Analysis and Due Diligence of New Business Ventures, Amer. Management Assoc., 2002.

Department of Mathematics

University of Rajasthan

Jaipur 302004, India

E-mail: gargmridula@gmail.com

School of Mathematics

University of Manchester

Manchester M13 9PL, UK

E-mail: mbbsssn2@manchester.ac.uk
Swami Keshvanand Institute of Technology, Management \& Gramothan Jaipur 302017, India E-mail: sangeeta11rc@gmail.com

Received on 11.9.2008;

revised version on 12.6.2009 
